\title{
Infectious Myositis of the Jaw Presenting as Trismus of Unknown Origin
}

\author{
Hee-Young Kim, Jin-Woo Chung
}

Department of Oral Medicine and Oral Diagnosis, School of Dentistry and Dental Research Institute, Seoul National University, Seoul, Korea

Received November 11, 2020 Revised December 17, 2020 Accepted December 18, 2020
Correspondence to:

Jin-Woo Chung

Department of Oral Medicine and Oral

Diagnosis, School of Dentistry and Dental

Research Institute, Seoul National University,

101 Daehak-ro, Jongno-gu, Seoul 03080,

Korea

Tel: +82-2-2072-3021

Fax: +82-2-744-9135

E-mail: jwchung@snu.ac.kr

https://orcid.org/0000-0003-3738-3386
Infectious myositis, an infection of the skeletal muscles, is a rare condition and potentially life-threatening if not detected and treated in the early stages. This clinical entity may arise from various pathogens, such as bacteria, fungi, parasites, and viruses. A propagation of contiguous infection, penetrating trauma, vascular insufficiency, or hematogenous spreading of microorganisms can cause infectious myositis. Though several cases have been reported in large muscle groups in the lower extremities, there are only a few reports on infectious myositis of the masticatory muscles. We report three cases of infectious myositis presenting jaw pain and trismus. Unlike a common head and neck infection caused by the spreading of odontogenic origin, the early diagnosis of infectious myositis was difficult because no specific lesion suspected to be the infection source was observed in the physical examination and the plain radiographs. Advanced imaging modalities such as computed tomography and magnetic resonance imaging, and laboratory evaluation is useful for the early detection of infectious myositis.

Key Words: Infection; Muscle; Myositis; Temporomandibular disorders; Trismus

\section{INTRODUCTION}

Trismus is a limitation of mandibular movement and mouth opening. The causes of trismus include spasm of the masticatory muscles and various other conditions. Typically, intra-articular factors such as internal derangement or ankylosis of temporomandibular joint (TMJ) and extra-articular factors such as infection of the orofacial region, and masticatory muscle disorders are possible causes of trismus [1].

Myositis is defined as the inflammation of skeletal muscles. The symptoms of myositis are pain, tenderness, swelling, and/or weakness, and can induce trismus when involved in masticatory muscles. Myositis is caused by infectious or non-infectious etiologies. Non-infectious etiologies include various clinical conditions, such as trauma, medication, autoimmune conditions, genetic disorders, endocrine disease, and electrolyte disturbances [2]. Meanwhile, infectious etiologies include various pathogens, such as bacteria, fungi, parasites, and viruses, causing infection of skeletal muscle [3]. Infectious myositis is a rare condition and potentially life-threatening if not detected and treated in the early stages [4]. Several cases have been reported in large muscle groups in the lower extremities such as piriformis and iliac muscle $[5,6]$. However, there are few reports on infectious myositis of the masticatory muscles.

In this paper, we present three cases of the infectious myositis in the masticatory muscles of unknown origin presented to the clinic with acute trismus. The study was approved by the Institutional Review Board of Seoul National University Dental Hospital (ERI20030). 


\section{CASE REPORT}

\section{Case 1}

A 38-year-old woman visited to the clinic with a 2-weeks history of limited mouth opening. She also complained of pain in the left pre-auricular area that had begun 2 months prior. She had been managed with analgesics and physical therapy at a dental clinic, but symptoms had worsened after stopping the drug. She had no significant premorbid medical history except for a history of cellulitis on the right side of jaw area 2 years prior. She reported a habit of jaw clenching and sleep bruxism.

Her mouth opening was limited to $23 \mathrm{~mm}$, and mandible deviated to the left side during mouth opening. She had pain on palpation of the left TMJ capsule and left masseter and temporalis muscles. Plain radiographs and cone-beam computed tomography showed flattening of the right mandibular condyle and erosive change in the cortical lining of the left mandibular condyle. An initial clinical diagnosis was made of anterior disc displacement without reduction and osteoarthritis of both TMJs, and myofascial pain of the masticatory muscles.

Although no fever or edema was observed in the clinical examination, she complained of swelling in her left jaw and was considered a previous history of cellulitis. A laboratory test, and magnetic resonance imaging (MRI) scan was performed to rule out infectious disease. Her laboratory data included a total white blood cell count (WBC) of 11,920 cells $/ \mu \mathrm{L}$ with $76.2 \%$ segmented neutrophils, the erythrocyte sedimentation rate (ESR) of $94 \mathrm{~mm} / \mathrm{h}$ and the C-reactive protein (CRP) of $13.31 \mathrm{mg} / \mathrm{dL}$. TMJ MRI revealed an anterior disc displacement on bilateral TMJs, and hyperintense T2 signal of the left masticatory muscles (masseter, temporalis, medial pterygoid, and lateral pterygoid muscles), which led to a final diagnosis of infectious myositis of the left masticatory muscle (Fig. 1). However, no obvious route of infection had been identified in the clinical examination or MRI.

She was hospitalized and received intravenous antibiotic therapy. At discharge, the laboratory data, including CRP level, had returned to normal range, and symptoms had relieved. However, she repeated the pain and swelling of the left jaw several times after discharge. Since management for degenerative osteoarthritis was also required, she had undergone conservative treatment of the temporomandibular disorder, including moist hot packs, exercise, stabilization splint therapy, and long-term non-steroidal antiinflammatory drugs (NSAIDs) administration. After that, the symptoms recovered and worsened repeatedly, and finally recovered to normal after 12 months.

\section{Case 2}

A healthy, 33-year-old man visited to the clinic with pain on the right TMJ and limited mouth opening that had begun suddenly 2 months prior. He had undergone physical therapy and stabilization splint therapy at a dental clinic for 2 months without relief, and his symptoms had worsened. He reported that the pain worsened in the morning after sleeping and waking up. He had no significant premorbid medical history.

On his first visit, he couldn’t open his mouth more than
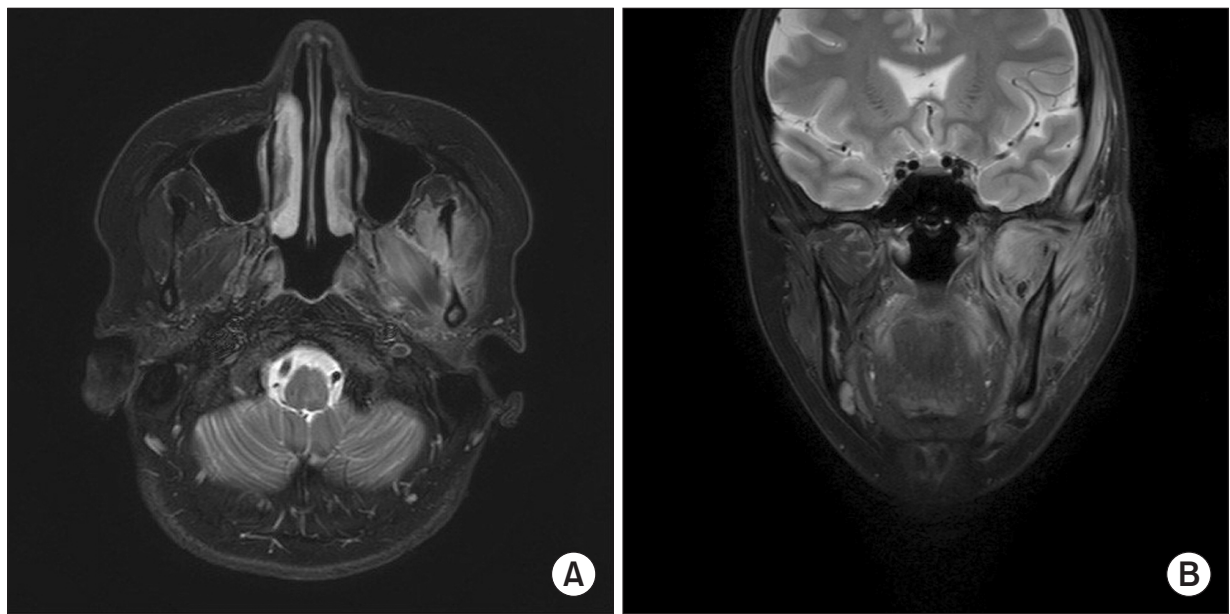

Fig. 1. Case 1. T2-weighted magnetic resonance images reveal high signal intensity in the left masticatory muscles. (A) Axial view. (B) Coronal view. 
$23 \mathrm{~mm}$. He had pain on palpation of both TMJ capsule and right temporalis muscle. Plain radiographs showed no bony change on either condyle. No sign of swelling or local heat was exhibited. An initial clinical diagnosis was made of ADD without reduction of TMJ and myositis of the temporalis muscles.

Less than 4 days later, the patient presented to the clinic reporting chills and markedly increased pain on the right facial area. Physical examination revealed a swollen and indurated region in the right buccal cheek to temporal area. Laboratory data included WBC of 11,880 cells/ $\mu \mathrm{L}$, absolute neutrophil count (ANC) of 8,233 cells/ $\mu \mathrm{L}$, and ESR of 58 $\mathrm{mm} / \mathrm{h}$. He was given oral antibiotics for possible cellulitis of the right masticatory muscle, and TMJ MRI was performed.

TMJ MRI showed an anterior disc displacement on bilateral TMJs, and hyperintense T2 signal of the right masticatory muscle (masseter, temporalis, medial pterygoid, and lateral pterygoid muscles) with swelling (Fig. 2A). No focal abscess or fluid collection was noted. Enhanced computed tomography (CT) scan was performed to find out an infection source. Still, no significant inflammatory changes in the jaw were confirmed, and swelling and enhancement of the right masticatory muscle were observed (Fig. 2B). A final diagnosis was ADD without reduction of both TMJs and infectious myositis of right masticatory muscles.

Though symptoms were temporarily relieved by oral administration of antibiotics (augmentin $625 \mathrm{mg}$ t.i.d), the pain and limited mouth opening worsened after a week. Therefore, he was hospitalized and intravenous antibiotic therapy (ceftriaxone) was performed for 2 weeks. He subsequently recovered and limited mouth opening, tenderness, and swelling of masticatory muscle were relieved at discharge.

After discharge, to prevent recurrence of symptoms and recover a normal range of mouth opening without jaw pain, he was managed with conservative treatment, including moist hot packs, exercise, stabilization splint therapy, and NSAIDs administration.

\section{Case 3}

A 64-year-old woman visited to the clinic with limited mouth opening, and pain on the right TMJ area while opening her mouth. She reported a habit of chewing primarily on the right side, and the symptom had begun after chewing dry squids 2 weeks prior. She had undergone acupuncture treatment at an oriental medicine clinic and prescribed analgesics at a dental clinic, but her symptoms had gradually worsened. She had no significant past medical history except for hypertension.

Her mouth opening was limited to $12 \mathrm{~mm}$. She had pain on palpation of the right TMJ capsule and right masseter muscle. Sign of swelling and local heat was exhibited on the right cheek area. Initial laboratory data included WBC of 21,870 cells $/ \mu \mathrm{L}$ with $78 \%$ segmented neutrophil, ANC of 17,059 cells/ $\mu \mathrm{L}$, and CRP of $17.62 \mathrm{mg} / \mathrm{dL}$. Plain radiographs showed degenerative bony change on the left side of the mandibular condyle, but no significant lesion of the jaw or tooth thought to be the cause of infection was identified. An initial clinical diagnosis was made of pterygomandibular space abscess. She was admitted to the department of

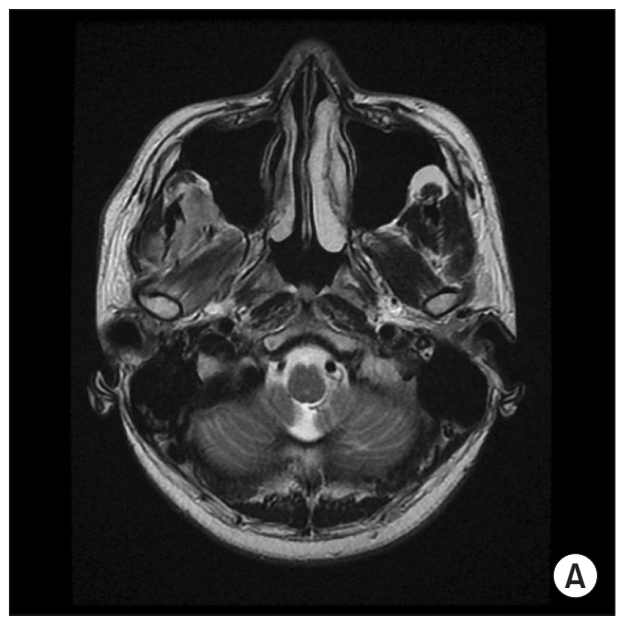

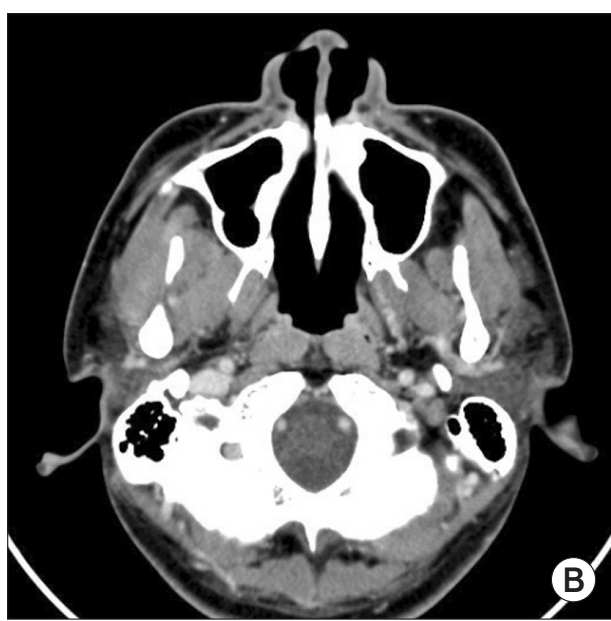

(1)
Fig. 2. Case 2. (A) An axial T2-weighted magnetic resonance image reveals high signal intensity in the right masticatory muscles. (B) An axial enhanced computed tomography image shows swelling and enhancement of right masticatory muscles. 
oral and maxillofacial surgery. On admission, she was febrile to $38.3^{\circ} \mathrm{C}$.

Enhanced CT scan revealed swelling of right masticatory muscle (lateral pterygoid, medial pterygoid, and masseter muscles). A fluid collection was found in the right lateral and medial pterygoid muscles (Fig. 3). Possible odontogenic factors were not identified. The final diagnosis was infectious myositis of the right masticatory muscles.

She underwent intravenous antibiotic therapy (amoxicillin-clavulanate, and metronidazole). Also, surgical drainage of the right medial and lateral pterygoid muscles was performed. A gram stain of the fluid revealed a few grampositive cocci. The abscess culture had grown 3 colonies of Klebsiella oxytoca, a gram-negative, rod-shaped, facultative anaerobe.

She was discharged after 2 weeks when the pain had subsided and laboratory data was changed into within a normal range. Approximately 4 months after initial symptoms, she was able to open her mouth to $45 \mathrm{~mm}$ without jaw pain.

\section{DISCUSSION}

Infectious myositis is defined as an infection of a skeletal muscle that arises from various pathogens. Infectious myositis due to bacteria is the most common, predominantly staphylococcal and streptococcal species [3,4]. A propagation of contiguous infection, penetrating trauma, vascular insufficiency, or hematogenous spreading of microorganisms can cause infectious myositis [3]. Certain hosts, those with immunocompromising conditions including HIV infection, malignancy, diabetes mellitus, and systemic sclerosis, have an increased risk of bacterial myositis $[7,8]$. However, recent articles have shown that infectious myositis can also affect immunocompetent individuals [8]. The patients in these three cases also had no identifiable immunodeficiency or predisposing factors.

Bacterial infection of the masticatory muscles is usually secondary to odontogenic infection progression such as pericoronitis or caries of a posterior tooth. Trismus and dysphagia were the most frequent presenting signs and symptoms when the severe odontogenic infection extended to the masticator and/or submandibular spaces $[9,10]$. However, in three cases no specific lesion suspected to be the infection source was observed in the physical examination and any radiographic imaging. The development of primary infection of muscle like this is suggested as a result of hematogenous spread. Since the healthy musculature is relatively resistant to infection, transient bacteremia without musculature damage is unlikely to cause bacterial myositis [11]. Bacterial myositis may occur in body sites that have been injured or compromised by a foreign body, trauma, ischemia, or surgery [12]. Overuse muscular injuries are also assumed to be a risk factor for the development of infectious myositis. There are reports of infectious myositis occurring in athletes, and they are at a slightly higher risk due to overuse injuries concurrent with asymptomatic episodes of bacteremia $[6,13]$. It has been suggested that repeated activation of masticatory muscles due to parafunction habits may activate the central mechanism and cause
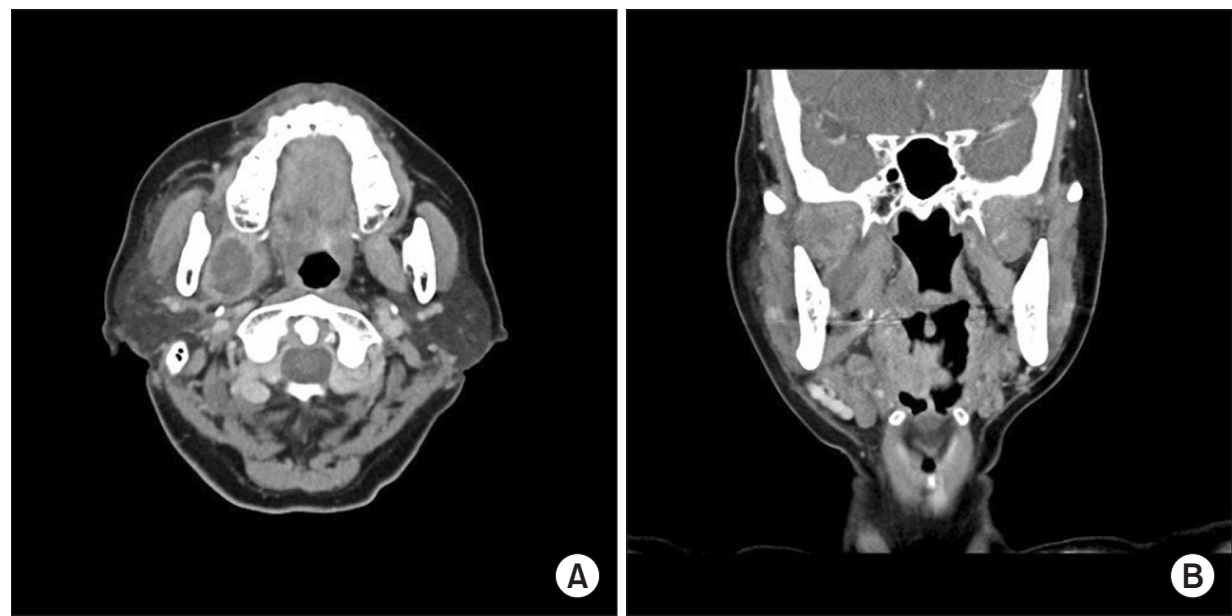

Fig. 3. Case 3. Enhanced computed tomography images show swelling and intramuscular abscess of the right lateral and medial pterygoid muscles. The airway space is narrowed because of the medial displacement of the oropharynx. (A) Axial view. (B) Coronal view. 
neurogenic inflammation of local muscles [14]. The three cases presented here exhibited that the patients had parafunctional habits such as clenching or a history of overuse of the masticatory muscles. Thus, muscle infections may occur when transient bacteremia seeds a site of compromised muscle due to overuse injuries. Since there are numerous resident bacteria in the oral cavity, and even without special dental procedures, daily activities such as eating, brushing, and flossing can also cause transient bacteriemia [15].

Infectious myositis can be treated successfully with intravenous administration of antibiotics and surgical drainage of abscesses [3,4]. Prognosis of infectious myositis is excellent except when the systemic spread of the infection leads to sepsis, shock, and multiorgan failure [16]. However, Infectious myositis presenting limited mouth opening can be misdiagnosed as other types of temporomandibular disorders when no characteristic findings are observed on the plain radiograph and symptoms such as swelling, warmth, and redness are ambiguous or develop later [17]. Thus, careful clinical examination and history taking should be performed. Moreover, the response of the treatment should be closely evaluated, and reassessment of the initial diagnosis is needed when the symptoms persist. It is important to use laboratory evaluation and advanced imaging modalities such as CT and MRI for the early diagnosis of infectious myositis. CT provides a good definition of the areas of involvement and guiding aspiration or drainage. MRI has better soft tissue contrast and early detection of the inflammatory process $[3,4]$.

\section{CONFLICT OF INTEREST}

No potential conflict of interest relevant to this article was reported.

\section{ORCID}

\author{
Hee-Young Kim \\ https://orcid.org/0000-0002-4788-3029 \\ Jin-Woo Chung \\ https://orcid.org/0000-0003-3738-3386
}

\section{REFERENCES}

1. Odell EW. Clinical problem solving in dentistry. 3rd ed. Edinburgh: Churchill Livingstone Elsevier; 2010. pp. 37-41.

2. Nagaraju K, Gladue HS, Lundberg IE. Inflammatory diseases of muscle and other myopathies. In: Firestein GS, Budd R, Gabriel SE, McInnes IB, O'Dell J, eds. Kelley and Firestein's textbook of rheumatology. 10th ed. Philadelphia: Elsevier; 2017. pp. 14611488.

3. Crum-Cianflone NF. Bacterial, fungal, parasitic, and viral myositis. Clin Microbiol Rev 2008;21:473-494.

4. Crum-Cianflone NF. Infection and musculoskeletal conditions: infectious myositis. Best Pract Res Clin Rheumatol 2006;20:10831097.

5. Martin A, Aftab S, Grewal US, Pampiglione T, Bracewell TG, Macerola AE. Infectious myositis of the iliacus muscle: an important differential in the unwell child with a limp. J Case Rep Images Surg 2015;1:17-20.

6. Chusid MJ, Hill WC, Bevan JA, Sty JR. Proteus pyomyositis of the piriformis muscle in a swimmer. Clin Infect Dis 1998;26:194195.

7. Schwartzman WA, Lambertus MW, Kennedy CA, Goetz MB. Staphylococcal pyomyositis in patients infected by the human immunodeficiency virus. Am J Med 1991;90:595-600.

8. Crum NF. Bacterial pyomyositis in the United States. Am J Med 2004;117:420-428.

9. Yonetsu K, Izumi M, Nakamura T. Deep facial infections of odontogenic origin: CT assessment of pathways of space involvement. AJNR Am J Neuroradiol 1998;19:123-128.

10. Kim KS. Facial pain induced by isolated lateral pterygoid pyomyositis misdiagnosed as trigeminal neuralgia. Muscle Nerve 2013;47:611-612.

11. Miyake H. Beitrage zur kenntnis der sogenannten myositis infectiosa. Mitt Grenzgeb Med Chir 1904;13:155-198.

12. Altrocchi PH. Spontaneous bacterial myositis. JAMA 1971;217: 819-820.

13. Koutures CG, Savoia M, Pedowitz RA. Staphylococcus aureus thigh pyomyositis in a collegiate swimmer. Clin J Sport Med 2000;10:297-299.

14. Kang JH, Huh KH, Kho HS. Non-infectious myositis of the lateral pterygoid muscle: a report of four cases. Int J Oral Maxillofac Surg 2015;44:226-228.

15. Forner L, Larsen T, Kilian M, Holmstrup P. Incidence of bacteremia after chewing, tooth brushing and scaling in individuals with periodontal inflammation. J Clin Periodontol 2006;33:401-407.

16. Chiedozi LC. Pyomyositis. Review of 205 cases in 112 patients. Am J Surg 1979;137:255-259.

17. Heo MS, An BM, Lee SS, Choi SC. Use of advanced imaging modalities for the differential diagnosis of pathoses mimicking temporomandibular disorders. Oral Surg Oral Med Oral Pathol Oral Radiol Endod 2003;96:630-638. 